\title{
KIN SELECTION IN THE ANNUAL PLANT IMPATIENS CAPENSIS
}

\author{
John K. KeLLY* \\ Department of Ecology and Evolution, University of Chicago, 1101 E. 57th Street, \\ Chicago, Illinois 60637
}

Submitted November 15, 1994; Revised September 27, 1995; Accepted October 11, 1995

\begin{abstract}
Kin selection occurs when phenotypic variation in a character or set of characters is heritable, spatially structured, and has differential fitness effects on neighboring individuals. Spatially structured, heritable variation has been found for many characters of the annual plant Impatiens capensis (the first two criteria). By manipulating plant growth by apical removal, I show that Impatiens fitness is strongly influenced by the phenotypes of neighboring plants, corroborating a previous study. A specific suite of phenotypes relating to plant architecture is consistently beneficial to neighboring plants. In addition, these manipulative experiments suggest that phenotypic plasticity may often impede purely observational field studies of kin selection in plant populations.
\end{abstract}

Plants often experience strong, fitness-determining interactions with neighboring individuals. Many studies have shown that variability in the growth, survival, or reproduction of individual plants is attributable to variation in the number or species identity of their neighbors (Harper 1977, chaps. 7-9). Variation in the phenotypic characteristics of neighbors may also have important effects on plant fitness (Horn 1971; Geber 1989; Stevens et al. 1995). The consequences of neighbor interactions for plant population dynamics and community structure are well appreciated (Harper 1977; Goldberg 1987; Pacala and Silander 1990). However, the implications of neighbor interactions for plant evolution have received less attention (Antonovics and Levin 1980; Griffing 1989).

The evolutionary consequences of neighbor interaction depend on the spatial genetic structure of a population. Because of limited seed and pollen dispersal, many plant populations exhibit a high degree of spatial genetic structure (Govindaraju 1988; Levin 1988). Neighboring conspecifics are often relatives; thus, fitness-determining interactions are expected to be heritable. Under these conditions, the standard methods for measuring natural selection on phenotypic variation (e.g., Lande and Arnold 1983) are inadequate. The fitness of a genetic variant depends not only on how it affects the survival and reproduction of the individual carrying it but also on how it affects the survival and reproduction of neighbors (Hamilton 1964a). When neighbor interactions occur, which is probably very frequently (Harper 1977), studies of natural selection based entirely on the relationship between individual phenotype and plant fitness are incomplete and

*E-mail: jkk1@midway.uchicago.edu. 
may be misleading (Wade 1980, 1985; Goodnight et al. 1992; Cheverud and Moore 1995).

Biologists have developed a large body of theory to describe natural circumstances in which the fitness of an organism depends on the genotypes of its neighbors. This theory includes inclusive fitness models (Hamilton 1964a; Grafen 1985; Queller 1992), family-structured models (Williams and Williams 1957; Wade 1980; Uyenoyama et al. 1981), intrademic group selection models (Hamilton 1975; D. S. Wilson 1975, 1980; Wade 1985), and quantitative genetics models (Willham 1963; Griffing 1967; Cheverud 1985). These models, which I collectively refer to as. "kin selection theory," have been applied to the evolution of social behavior in a wide range of animal taxa, especially insects (Hamilton $1964 b$; E. O. Wilson 1975; Breden and Wade 1989; McCauley 1994). However, with a few exceptions (Allard and Adams 1969; Goodnight 1985; Griffing 1989; Stevens et al. 1995), kin selection has not been considered for plant populations.

I suggest that three basic elements are necessary to demonstrate the action of kin selection on a character or set of characters in a natural population. First, there must be heritable variation for the character(s) within the population. This is a requisite for evolution by all forms of natural selection and is not limited to kin selection. Second, this variation must be spatially structured within the population so that there is a genetic correlation between neighboring organisms. The evolutionary importance of neighbor effects relative to individual effects on survival and reproduction is determined by the magnitude of this correlation (Hamilton 1964a). Third, variation in the character(s) of neighboring plants must have effects on the survival or reproduction of focal individuals.

Previous studies suggest that these three criteria may be satisfied for the annual plant species Impatiens capensis. Heritable variation has been found for phenological, morphological, and performance traits in natural populations of Impatiens (Schemske 1984; Mitchell-Olds and Bergelson 1990a; Argyres and Schmitt 1991; Dudley and Schmitt 1995). In addition, this variation has a distinct spatial structure (Schemske 1984; Knight and Waller 1987; Schoen and Latta 1989; Argyres and Schmitt 1991). Limited gene flow due to frequent self-fertilization and limited seed dispersal distance allows genetic differentiation of Impatiens patches (Schmitt et al. 1985). Knight and Waller (1987) estimated $F$ statistics from allozyme variation: mean $F_{\mathrm{IS}}=0.57, F_{\mathrm{ST}}=0.46$. Relatedness can be calculated directly from these $F$ statistics (Wright 1968) by the formula $r=2 F_{\mathrm{ST}} /\left(1+F_{\mathrm{IT}}\right.$ ), where $F_{\text {IT }}=1-\left(1-F_{\text {IS }}\right)\left(1-F_{\mathrm{ST}}\right)$. Applying this formula, we obtain an estimate of $r=2(0.46) /(1+0.71)=0.54$. This suggests that the average relatedness of plants within the same Impatiens patch is about the same as full siblings in a panmictic population. This high level of interpatch variation is also observed for quantitative variation of Impatiens (Schemske 1984; Dudley and Schmitt 1995).

A recent study by Stevens et al. (1995) suggests that the third criterion for kin selection may also be satisfied in $I$. capensis. Stevens et al. took a series of phenotypic measurements on plants in local neighborhoods $(0.25-\mathrm{m}$ radius circles $)$ within a large natural patch of Impatiens and monitored their survival and reproduction. They used the multiple-regression method of contextual analysis (Heisler 
and Damuth 1987) to partition the effects of individual plant characters from the effects of local group characters in determining plant fitness. They found significantly nonzero partial regression coefficients of fitness onto group level traits, which suggests the operation of group-level selection (Goodnight et al. 1992).

This article describes an experimental study of neighbor interactions in 1 . capensis. I manipulated the phenotypes of plants and their neighbors by removing the apical tip from selected plants. The intended effect of manipulation was to remove the apical meristem, releasing plant growth from apical dominance and thus generating relatively greater investment in lateral growth. Plant architecture mediates competition among neighbors for access to light (Schmitt et al. 1987; Geber 1989; Weiner et al. 1990). I monitored the fitness consequences of these manipulations over $2 \mathrm{yr}$ in four different populations. The purpose of these experimental manipulations was to determine whether the correlations between neighbor phenotypes and plant fitness described by Stevens et al. (1995) have a causal basis. The results from these experiments provide direct evidence that the third criterion for kin selection is satisfied in populations of 1 . capensis.

\section{STUDY SPECIES}

Impatiens capensis, or jewelweed, is a common North American annual plant in the family Balsaminaceae. It tends to occupy shady, moist habitats often along waterways. It is an annual, and the complete reproductive success of an individual can be assessed over a single year. Impatiens reproduce continuously over a period of several months, but the fruit pedicels persist on the plant, which allows accurate estimation of total fruit production (Stevens et al. 1995). Impatiens capensis and with its close relative Impatiens pallida have been the focus of numerous studies examining growth and competition (Waller 1985; Mitchell-Olds 1987; Schmitt et al. 1987; Weiner et al. 1990; Dudley and Schmitt 1996), population structure (Schemske 1984; Schmitt et al. 1985; Knight and Waller 1987), and natural selection (Schemske 1984; Stewart and Schoen 1987; Mitchell-Olds and Bergelson 1990b; Stevens et al. 1995).

\section{STUDY SITES AND METHODS}

In 1993, manipulations were conducted in three natural patches of Impatiens, denoted p1, p2, and p3, respectively. In 1994, manipulations were conducted in p1 and a new patch, p4. Population p1 is located in the Kellogg Bird Sanctuary, Kalamazoo County, Michigan. Populations p2, p3, and p4 are located in the Kellogg Experimental Forest, Kalamazoo County, Michigan. Population p1 is a triangular patch approximately $15 \mathrm{~m}$ long and $8 \mathrm{~m}$ wide (at its widest) growing around a tree fall, adjacent to Wintergreen Lake. It is a dense, largely monospecific patch of Impatiens exposed to direct sunlight. Populations p2 and p3 are about $70 \mathrm{~m}$ apart on a flood plain adjacent to the Augusta River. Population p2 is approximately $20 \times 40 \mathrm{~m}$ with intermediate exposure to direct sunlight. Population p3 is approximately $10 \times 10 \mathrm{~m}$ under a dense canopy that absorbs most 
direct sunlight. Impatiens density is substantially lower in p2 and p3 than in p1, and the abundance of other species is correspondingly higher. Population $\mathrm{p} 4$ is a large $(40 \times 30 \mathrm{~m})$, intermediate-density patch of Impatiens growing under a stand of conifers that absorb most direct sunlight.

I manipulated the growth and allocation patterns of Impatiens in the field by removing the apical tip from plants in the middle of the growing season. I removed approximately $1 \mathrm{~cm}$ from the top of the main stem with one or two immature leaves. Twenty focal plants were haphazardly selected and flagged within each of $\mathrm{p} 1, \mathrm{p} 2$, and $\mathrm{p} 3$ on July 19,1993 . At least $1 \mathrm{~m}$ separated focal plants. Ten plants in each population were randomly selected for treatment, while the other 10 were left untreated as controls. The apical tips were snipped from all neighboring Impatiens within $30 \mathrm{~cm}$ of a treatment focal plant. Under the null hypothesis that plant fitness is independent of the characteristics of neighboring plants, neighbor manipulation should have no effect on the performance of focal plants. All focal plants were left unmanipulated in 1993.

All focal plants and their neighbors within $20 \mathrm{~cm}$ were harvested between September 9 and September 16, 1993. (In 1993, a smaller radius, $20 \mathrm{~cm}$, was adopted for sampling rather than the $30 \mathrm{~cm}$ for manipulation due to personnel constraints.) Focal plants were noted as alive or dead, and live plants were measured for height, number of leaves on opposite branches, total number of leaves, and number of fruit pedicels. I was usually able to assess the fruit production of plants that died after the start of reproduction because their stem and branches remained within the patch. These plants were scored as having survived until reproduction.

Impatiens capensis shifts from opposite to alternate branching as it matures. A quantity that I denote as the leaf ratio was obtained by dividing the number of leaves on opposite branches by the total number of leaves on the plant. The leaf ratio measures the relative allocation of photosynthetic tissue to lower branches (opposite) versus upper branches (alternate). Higher values of leaf ratio indicate a bottom-heavy or "bushy" phenotype.

The total number of conspecific neighbors (within $20 \mathrm{~cm}$ ) was counted, and the height and leaf characters were determined for each in 1993. One focal plant in p1 and one focal plant in p3 were destroyed by artificial agents and were eliminated from the analysis of results. Neighbor measurements were not taken from two of the remaining groups in $\mathrm{p} 1$, and these cases are eliminated from analyses requiring these measurements.

In 1994, both focal plants and neighbors were subject to manipulation. The focal plants in each population were evenly divided into four experimental treatments: focal manipulated/neighbors manipulated, focal manipulated/neighbors unmanipulated, focal unmanipulated/neighbors manipulated, and focal unmanipulated/neighbors unmanipulated. Twenty-four focal plants were flagged in $\mathrm{p} 1$, and 80 were flagged in $\mathrm{p} 4$. The manipulations were performed on July 10 in p1. The plants were manipulated twice in p4, first on June $27-28$ and then again on July 14-15. For the second round of manipulation in $\mathrm{p} 4$, I removed the tip from the branch in the most apical position. All focal plants and neighbors within $30 \mathrm{~cm}$ 
were harvested from p1 on September 9-10 and from p4 on September 11-13. The total number of conspecific neighbors was counted, but only the focal plant and two randomly selected neighbors were measured. Each focal plant was noted as alive or dead, and live plants were measured for height, number of leaves on opposite branches, total number of leaves, and number of fruit pedicels. The height on the central axis of the plant from which each lateral branch grew was also noted, as was the distribution of leaves across these branches. The same set of measurements, except for the number of pedicels, was made on the two neighbors selected near each focal plant.

I calculated a second plant architecture statistic, $\theta$, from the more intensive measurements made in 1994. Theta is equal to the average vertical position of a leaf along the central axis of the plant:

$$
\theta=\sum_{i}\left(\frac{H t_{i}}{H t}\right)\left(\frac{L_{i}}{L}\right),
$$

where $H t_{i}$ is the height along the plant stem from which the $i$ th branch extends, $H t$ is the total height, $L_{i}$ is the number of leaves on the $i$ th branch, $L$ is the total number of leaves, and the summation is taken over all branches. Theta is a measure of the vertical distribution of leaves (e.g., Weiner et al. 1990). Its value ranges from zero to one, with lower values of $\theta$ indicating relatively greater leaf production on lower branches.

\section{ANALYSIS}

The 1993 experiments were analyzed to determine whether there were significant differences in performance between focal plants surrounded by manipulated neighbors (denoted $\mathrm{M}$ ) and focal plants surrounded by unmanipulated neighbors (denoted C). Chi-square contingency table tests were performed on survivorship data. ANOVA was used to detect treatment effects on continuous phenotypic measures of both neighbors and focal plants and fruit production of focal plants. Fruit production of surviving focal plants was square-root transformed to improve normality. The product of survival (zero or one) and fruit production, denoted $W$, was also calculated. The distribution of $W$ will generally be nonnormal. For this reason, $t$-tests allowing unequal variance within factors were used to assess treatment effects on $W$ because, with sufficient sample sizes, this test is robust to nonnormal data (Sokal and Rohlf 1981).

The 1994 experiments were factorial designs with two factors: focal treatment and neighbor treatment. Chi-square contingency table tests were performed separately for each factor to detect an effect of either focal or neighbor treatment on survival. Separate $t$-tests were also performed to detect each type of treatment effect on $W$. Two-way ANOVAs were applied to the continuous phenotypic values and fruit production of focal plants. The ANOVAs allow us to evaluate interactions between focal and neighbor treatments. 


\section{RESULTS}

\section{3}

The results can be broken into two parts: the effects of manipulation on neighbor phenotypes, and the subsequent effects of variable neighbor phenotype on focal plant phenotype and fitness components. In $\mathrm{p} 1$, manipulation of neighbors resulted in significantly lower density of adult neighbors, a greater mean neighbor height, and a greater mean neighbor leaf number (table 1). Manipulation caused a substantial, but nonsignificant, increase in the mean leaf ratio of neighbors. Manipulated and unmanipulated focal plants did not differ significantly in survivorship, but there was a large positive effect of neighbor manipulation on fruit production $(F=9.76$, df $=1,16, P<.007)$ and $W\left(t_{11.9}=-2.55, P<.03\right)$ (fig. 1).

Manipulation had a minimal effect on neighbor phenotypes in populations $\mathrm{p} 2$ and $\mathrm{p} 3$. In $\mathrm{p} 2$, manipulation significantly affected leaf ratio of neighbors, but none of the other characters (table 1). In p3, manipulation had no significant effect on neighbor phenotypes (table 1). There were no significant differences among focal plants in either population.

\section{4}

The effects of manipulation on neighbor phenotypes in p1 in 1994 differed between the set of groups with manipulated focal plants and the set of groups with unmanipulated focal plants. The set of groups with unmanipulated focal plants was a replication of the 1993 experiment in this population. The results in this set of groups were similar to those observed the previous year: manipulation produced "bushier" neighbors; that is, they were, on average, taller with more leaves, a higher leaf ratio, and lower $\theta$ (table 2 ; open squares in fig. $2 B$ ). As in the previous year, this subset of the focal plants did better when surrounded by manipulated neighbors than by unmanipulated neighbors (open squares in fig. $2 A$ ), although this difference was not statistically significant, possibly because of the smaller sample size (12 vs. 19).

The effects of neighbor manipulation were reversed in groups with manipulated focal plants (table 2). Here, manipulated neighbors had fewer leaves, lower leaf ratios, and a higher average $\theta$ (solid circles in fig. $2 B$ ). Thus, in this set of groups, the control neighbors were relatively bushier than the manipulated neighbors. Furthermore, reproductive output of focal plants was greater near unmanipulated neighbors (solid circles in fig. $2 A$ ). This reversal of neighbor treatment effect on focal plant fruit production across focal plant treatments yielded a marginally significant interaction between the focal and neighbor factors $(F=3.37$, $\mathrm{df}=$ $1,19, P<.08)$.

In $\mathrm{p} 4$, neighbor manipulation consistently increased the mean number of leaves per neighbor, increased the leaf ratio, and reduced $\theta$ (table 3 ). Survivorship and reproduction of both manipulated and unmanipulated focal plants were greater near manipulated neighbors. Survival $\times$ fruit production $(W)$ of focal plants near manipulated neighbors exceeded twice $W$ of focal plants near unmanipulated neighbors (fig. $3 ; t_{63.6}=-2.33, P<.02$ ). Unmanipulated focal plants had greater 


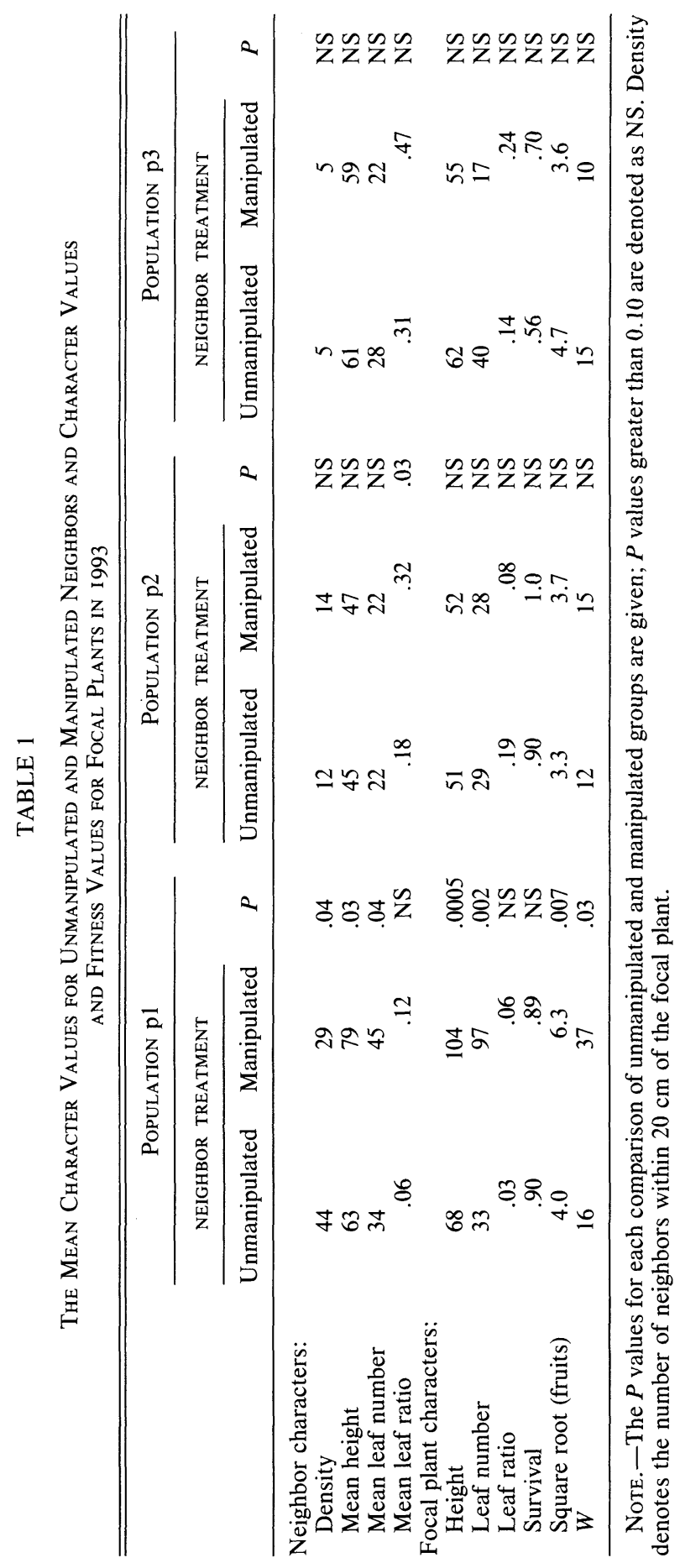




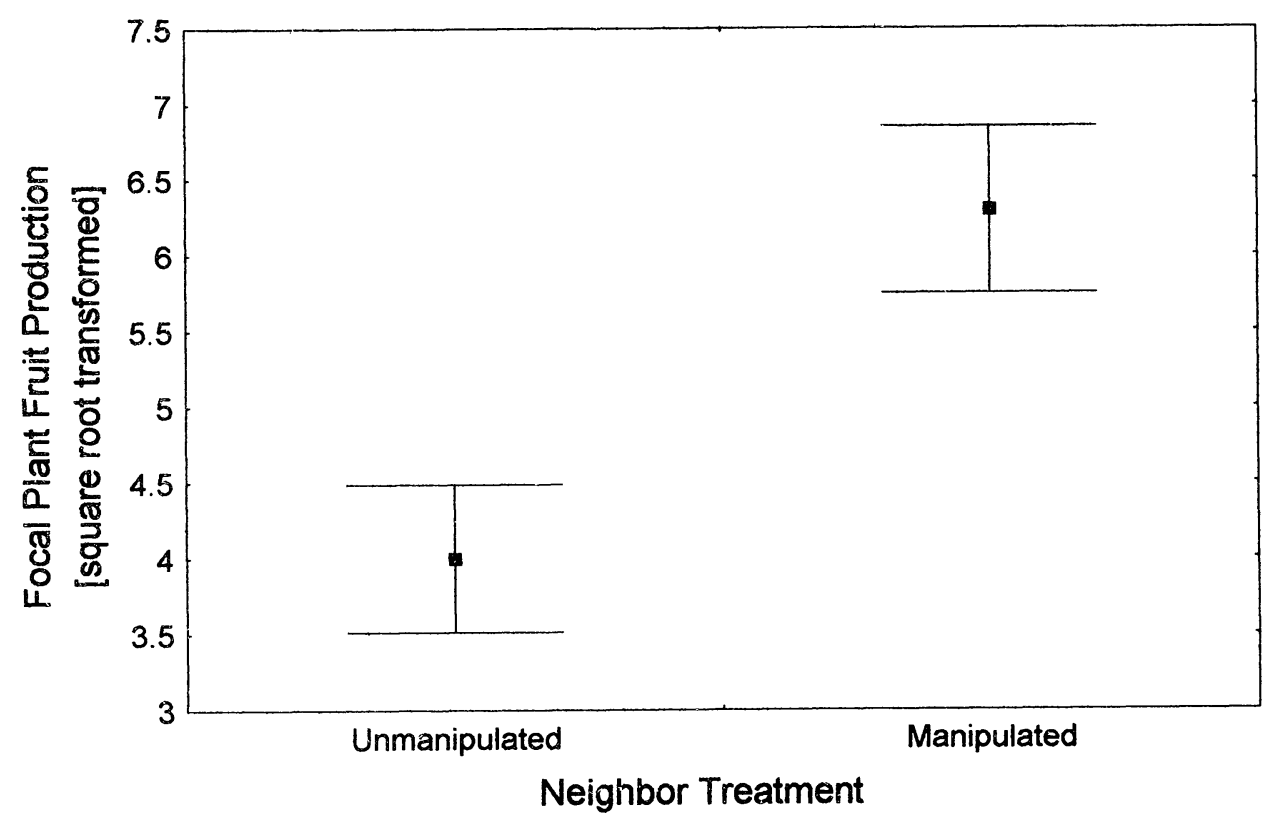

FIG. 1.-The mean and SE of fruit production (square-root transformed) of focal plants among unmanipulated and manipulated neighbors in p1, 1993.

average seed production than manipulated focal plants in $\mathrm{p} 4$ (table $3 ; F=3.58$, df $=1,39, P<.07)$.

An important feature of these results is that the manipulations of neighbor phenotypes were well within the natural range of variation for the characters monitored. For example, manipulation increased the mean number of leaves per neighbor from 34 to 45 in p1 in 1993 (table 1). However, this induced increase in mean neighbor leaf number in $\mathrm{p} 1,1993$, is less than the mean neighbor leaf number in unmanipulated groups (62) in p4, 1994 (table 3). Thus, these results are not an artifact of extreme experimental conditions.

A simple test of the null hypothesis that focal plant fitness is independent of the characteristics of neighboring plants can be obtained for the entire series of experiments (thus avoiding any problem of multiple tests) by treating each experiment as a single data point. In the absence of kin selection, we expect no difference in mean survival or reproduction between focal plants with unmanipulated neighbors and those with manipulated neighbors within an experiment. Any difference between means, denoted $W_{M}-W_{C}$, would have to be attributed to random variation and would be unrelated to the effect of manipulation on neighbor phenotypes within the experiment. In contrast, under kin selection, we expect the fitness difference between focal plants with manipulated and unmanipulated neighbors to be directly related to how strongly manipulation alters the phenotypes of neighbors.

An application of this test to these experiments reveals that the magnitude 


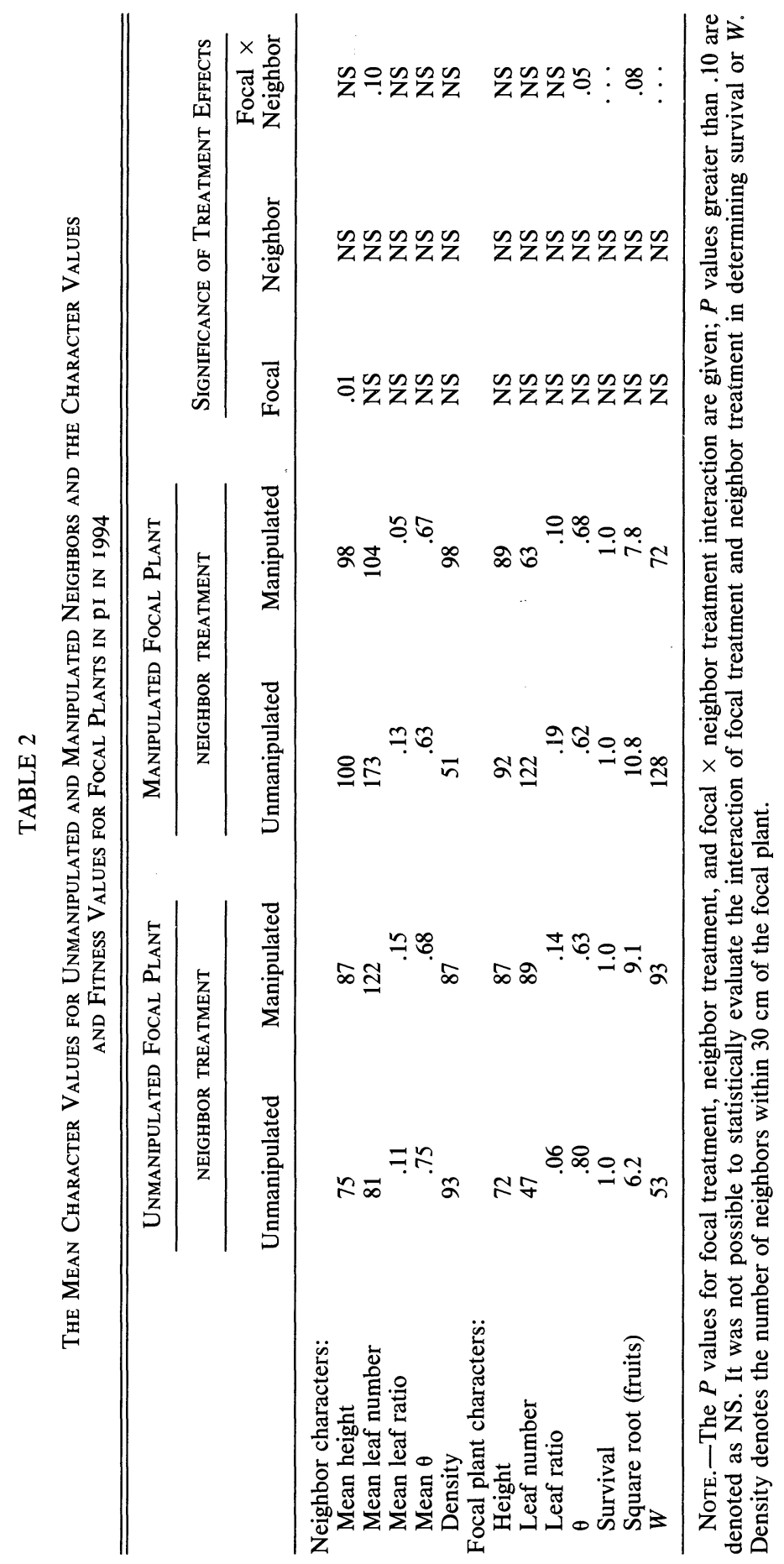




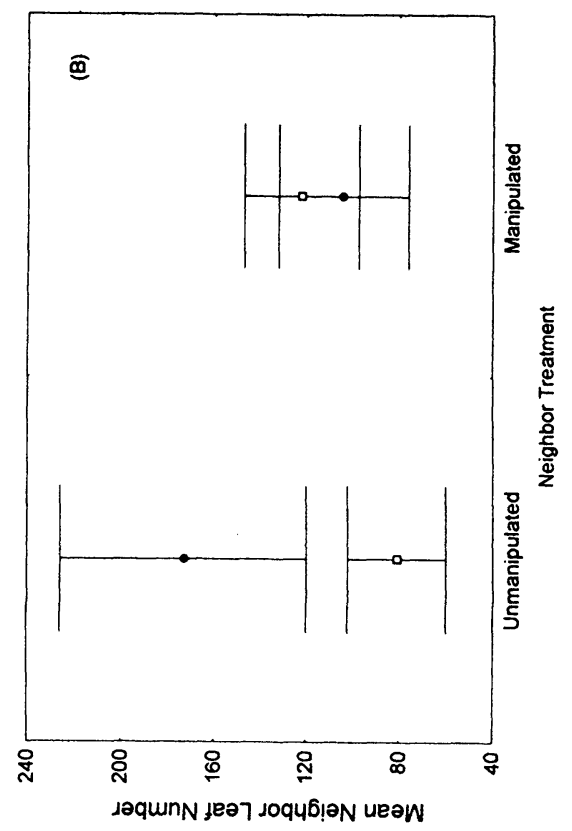

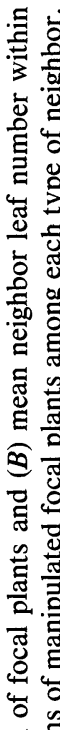

รี

:

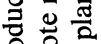

పే

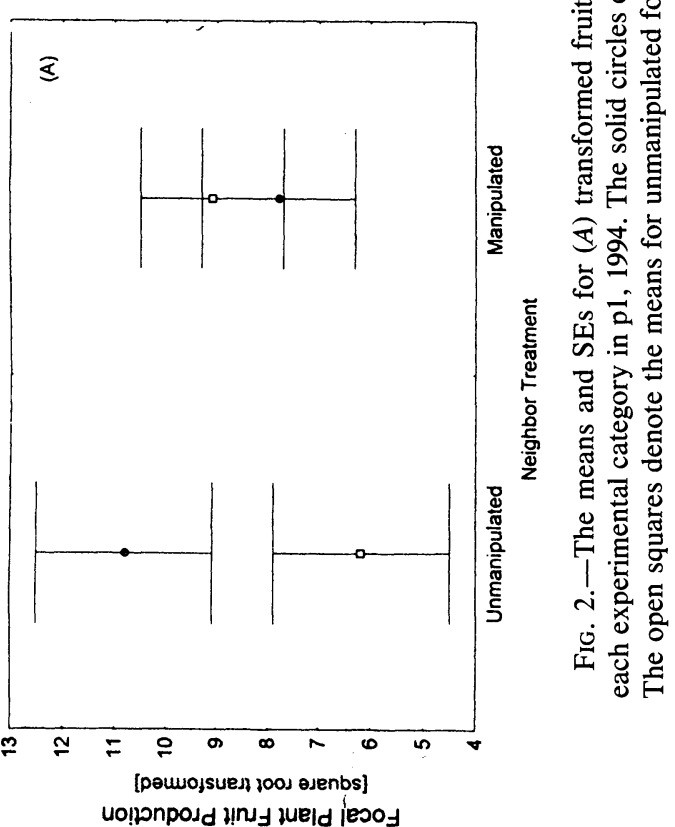

908 


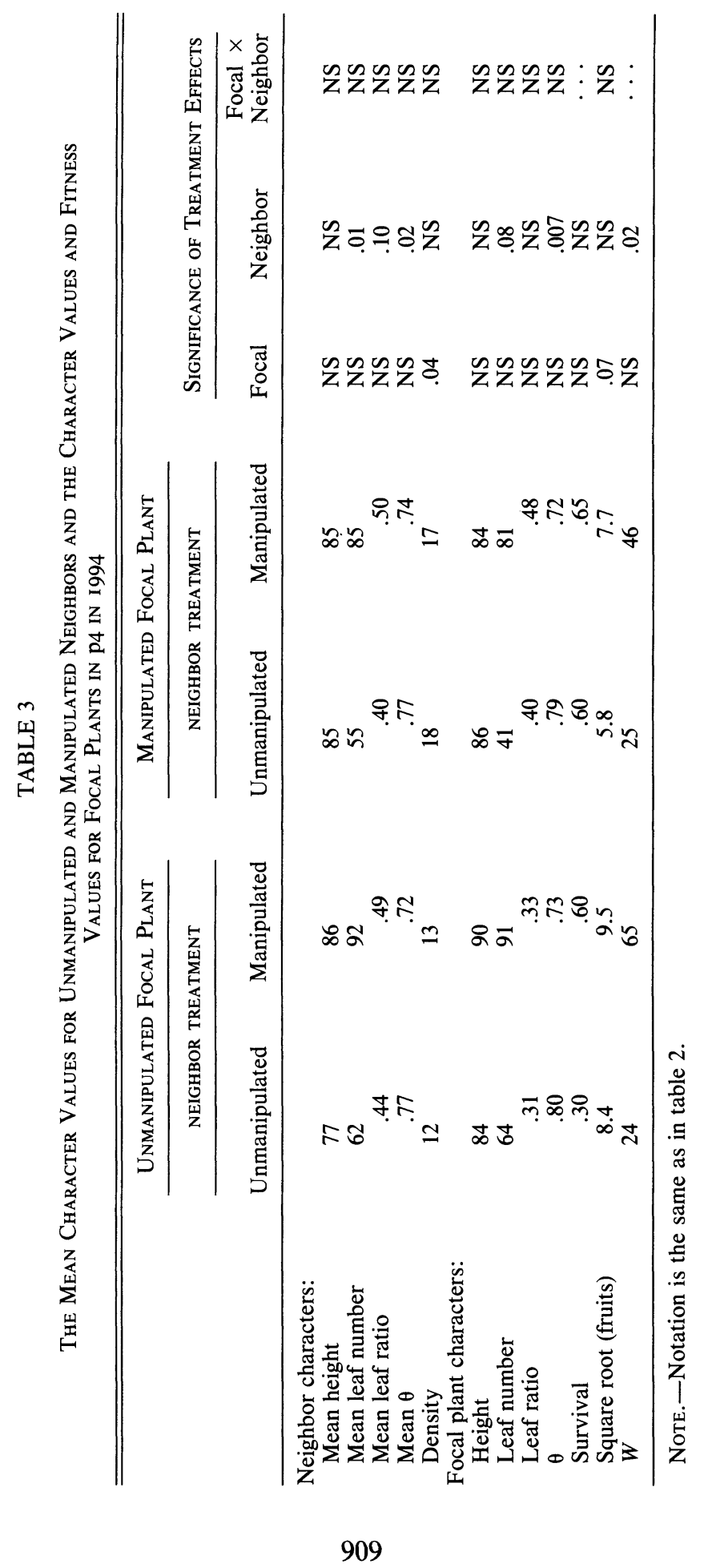




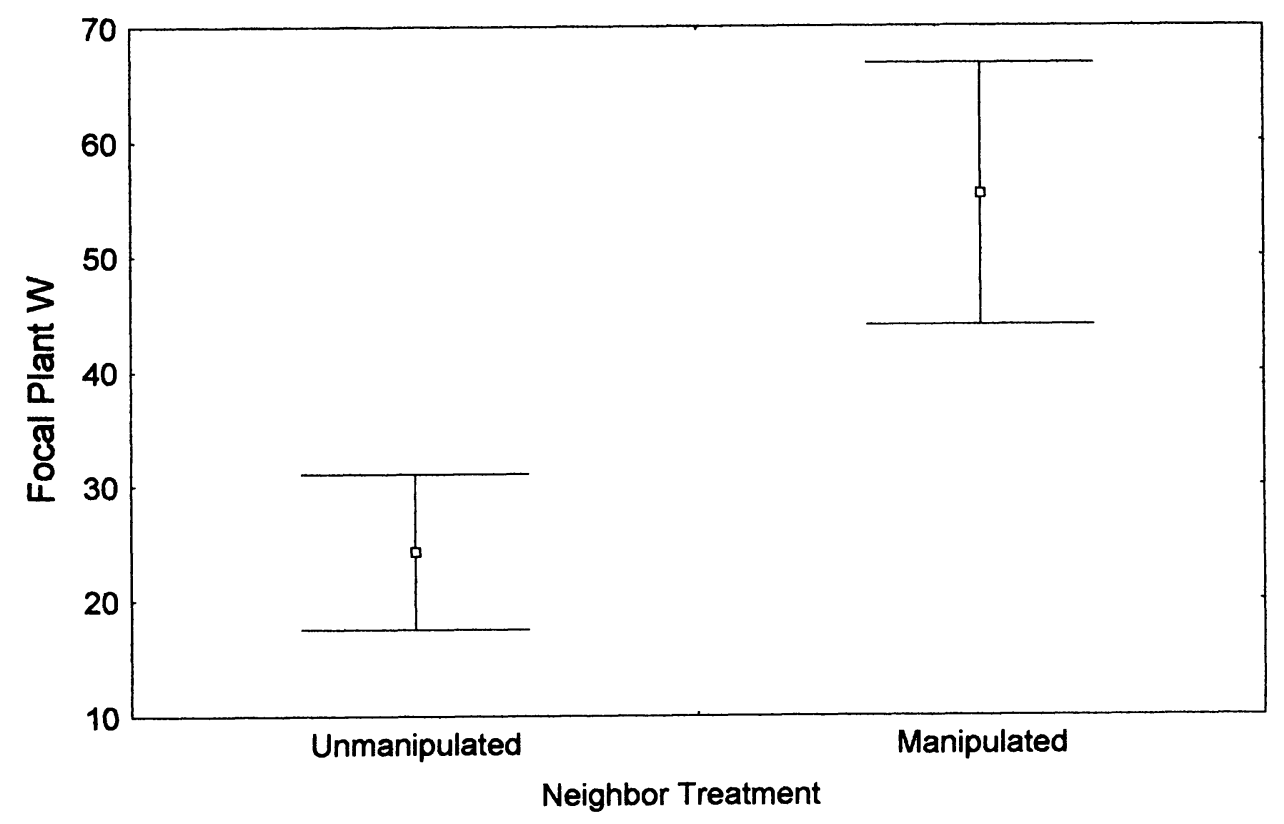

FIG. 3.-The mean and SE of $W$ of focal plants among unmanipulated and manipulated neighbors in p4, 1994 (averaging over both classes of focal plants).

of the effect that neighbor manipulation has on focal plant fitness is strongly correlated with the magnitude of the effect that manipulation has on the phenotypes of neighboring plants (fig. $4 ; r=0.98, P=.0001$ ). (Each 1994 experiment was split into two comparisons: unmanipulated focal plants with unmanipulated vs. manipulated neighbors, and manipulated focal plants with unmanipulated vs. manipulated neighbors.) In each case that manipulation substantially altered the mean number of leaves per neighbor (e.g., p4 in 1994), large effects on $W$ were observed. In experiments testing whether manipulation had weak effects on the mean number of leaves per neighbor (e.g., p2 and p3 in 1993), the response of focal plants was minimal. The correlation remains significant $(r=0.92, P=$ .009) even if the point in the lower left corner is eliminated (p1, 1994, focal plants manipulated).

\section{DISCUSSION}

\section{Neighbor Interaction and the Measurement of Selection}

In recent years, a large number of studies have measured natural selection in plant populations (e.g., Kalisz 1986; Stratton 1992 and references therein). These studies generally employ regression methods in which components of fitness are predicted as a function of the individual multivariate phenotype (Lande and Arnold 1983; Arnold and Wade 1984a, 1984b)! This methodology provides metrics of natural selection that are directly related to evolutionary change under a spe- 


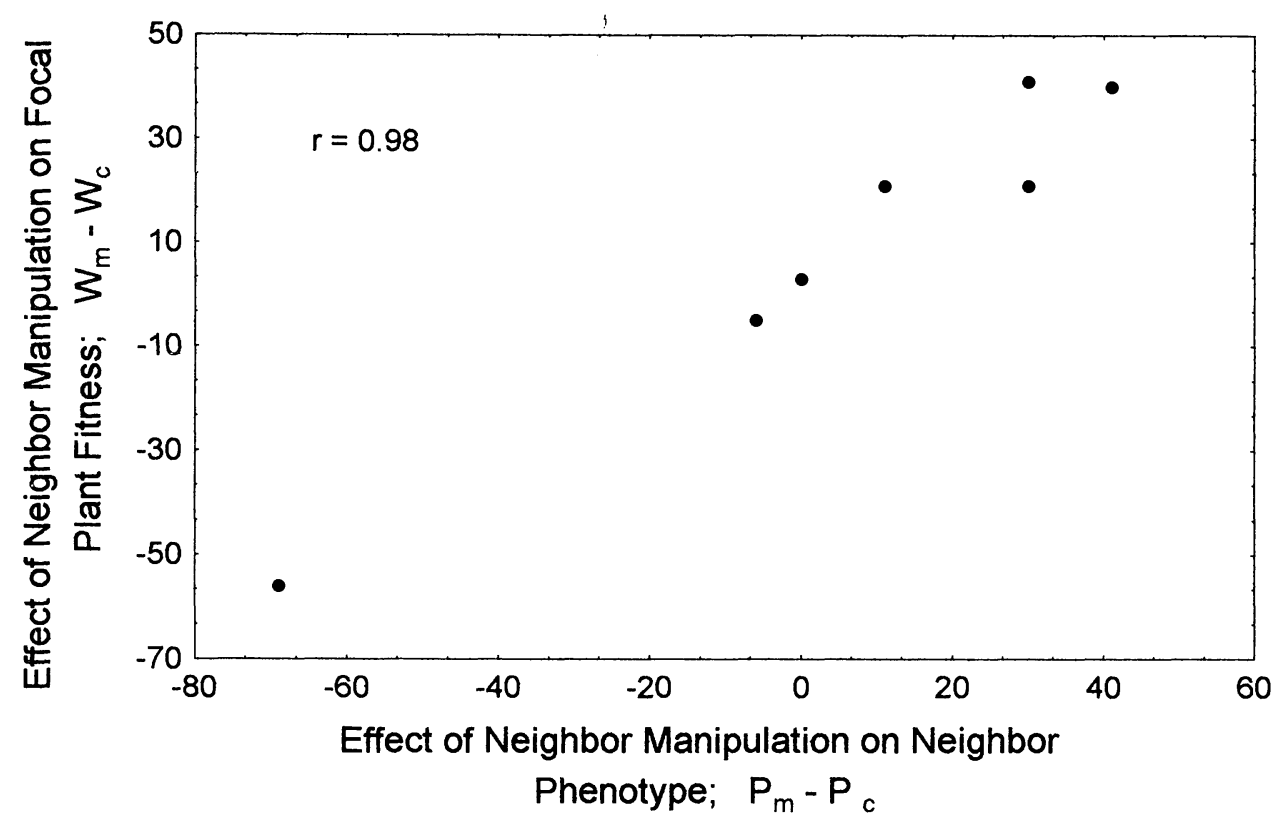

FIG. 4.-The relationship between the difference in $W$ between focal plants surrounded by unmanipulated and manipulated neighbors $\left(W_{\mathrm{m}}-W_{\mathrm{c}}\right)$ within an experiment and the difference in mean leaf number between unmanipulated and manipulated neighbors $\left(P_{\mathrm{m}}-\right.$ $P_{\mathrm{c}}$ ) in that experiment.

cific set of assumptions. These assumptions include a quantitative genetic model of inheritance in which the phenotype of an individual-and, ultimately, its fitness-is determined by its individual genotypic value plus a random error (Lande 1979).

Kin selection violates an important assumption of this quantitative genetic model. The fitness of an individual depends not only on its own genotypic value but also on the genotypic values of its neighbors (the first and third criteria). The latter effect cannot be dismissed as "environmental noise" when there is genetic relatedness among neighbors (the second criterion). In quantitative genetic terminology, neighbor selection implies a genotype $\times$ environment covariance for fitness (Falconer 1981). When this covariance is significant, selection gradients based on individual phenotypic values are suspect.

The evolutionary consequences of kin selection can also be illustrated with a path diagram (fig. 5; see also Willham 1963; Lynch 1987; Cheverud and Moore 1995). Classic individual selection is represented by paths $a, b, e_{1}$, and $e_{2}$. Variation in plant phenotype is determined in part by plant genotype (path $a$ ), and this variation is associated with differential survival and reproduction (path $b$ ). The covariance between focal plant genotypic value and focal plant fitness determines evolutionary change (Robertson 1966; Price 1970). In the absence of kin selection, this covariance is determined by paths $a$ and $b$ and the error terms, $e_{1}$ and $e_{2}$. 


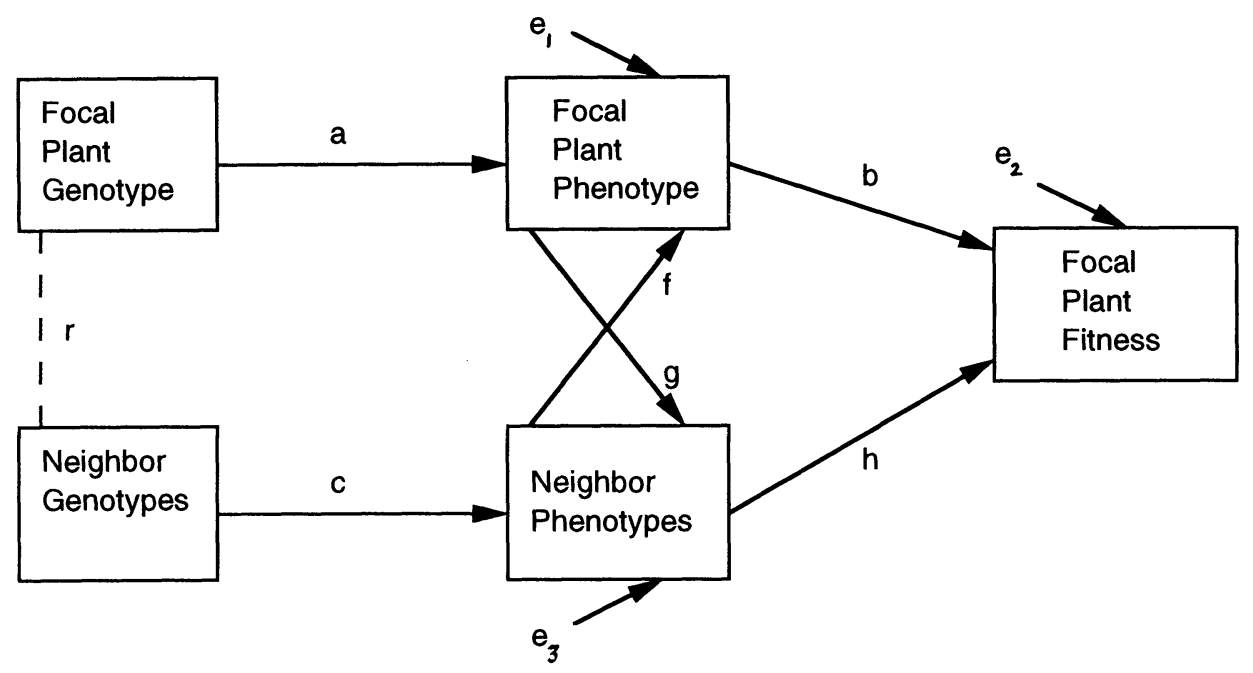

FIG. 5.-A path diagram illustrating the consequences of neighbor interaction for response to selection. Paths are described in the text.

The three components of kin selection-heritable variation (represented by paths $a$ and $c$ in fig. 5), neighbor relatedness (link $r$ ), and fitness dependence on neighbor phenotypes (path $h$ or path $f$ to $b$ ) -imply several additional pathways between focal plant genotype and focal plant fitness. When these links and paths have nonzero values, individual selection is only one component of evolutionary change (Hamilton 1975; Wade 1985). The covariance between focal plant genotype and fitness includes a group or neighbor component, which must be included to correctly estimate the genetic response to selection.

Several models have been developed to expand quantitative genetic methods for measuring selection to accommodate neighbor interactions (Willham 1963; Cheverud 1985; Heisler and Damuth 1987; Lynch 1987). While these models differ in detail, they all indicate that evolutionary change will depend on factors that are usually ignored in studies of natural selection in plant populations. These factors include aspects of population structure such as the dispersal, breeding biology, and population density regulation (Antonovics and Levin 1980; Wilson 1980; Wade and Breden 1987; Kelly 1994a). A greater range of evolutionary outcomes must also be considered. Specifically, the evolution of group-advantageous or neighbor-beneficial characters is a possible novel evolutionary consequence of kin selection.

\section{Kin Selection in Impatiens}

The purpose of these experiments was to determine the extent to which Impatiens fitness depends on the characteristics of neighboring plants. This relationship, which I have referred to as the third criterion for demonstrating kin selection, was examined thorough manipulations of plant growth. The effect of neighbor manipulation on focal plant fitness varied among populations and years. 
Neighbor manipulation had a large and statistically significant effect on focal plant fitness in experiments p1, 1993, and p4, 1994. It had important but complicated effects in p1, 1994. Neighbor manipulation had minimal effects on focal plant fitness in p2, 1993, and p3, 1993. However, in each case that manipulation significantly affected the mean phenotypes of neighboring plants, a large response was observed in focal plant fitness components (fig. 4). When combined with the previous study by Stevens et al. (1995), these experiments provide strong evidence that the third criterion for kin selection is satisfied in at least some natural populations of Impatiens capensis.

Focal plants consistently performed better when their neighbors had more leaves, a higher leaf ratio, and lower values of $\theta$. I refer to this suite of characteristics as the "bushy phenotype.' In p1, 1993, and p4, 1994, neighbor manipulation generated bushy neighbors. Consequently, focal plants performed better near manipulated neighbors. In p1,1994, manipulation of neighbors increased the bushiness of neighbors around unmanipulated focal plants and reduced the bushiness of neighbors around manipulated focal plants. The relative fitness of focal plants among the two groups reversed in parallel with the differing effects of treatment on the neighbors (cf. fig. $2 A$ and $B$ ). In addition to the fitness response to neighbor manipulation, phenotypic changes in focal plants were also observed. Focal plants developed a relatively bushy phenotype when their neighbors were bushy.

One component of the bushy phenotype, adult leaf number, is strong correlated with fruit production among focal plants $(r=0.95$ among focal plants in 1994). If this relationship holds for both focal plants and neighbors, it suggests that the mean fitness of groups of bushy plants (focal and neighbors) is substantially higher than the mean fitness of nonbushy groups. Significant differences in mean fitness among groups with different phenotypic compositions are an expected consequence of kin selection.

These results, when combined with previous studies demonstrating character heritability and spatial genetic structure in I. capensis, suggest a simple qualitative conclusion. The inclusive fitness of a genetic variant affecting morphological traits of Impatiens may be determined, to an important extent, by interactions with genetically related neighboring plants. Stating the same conclusion from a quantitative genetic perspective, the covariance between the genotypic values of morphological characters and fitness will often depend on causal pathways involving neighbor genotypes and phenotypes (paths $r, c, f$, and $h$ in fig. 5).

These results also suggest a number of avenues for further research. First, the manipulations in these experiments simultaneously affected a broad range of plant characters. It is not possible to tell which changes in neighbor phenotype were responsible for changes in focal plant survival and fruit production. Precise manipulations of specific phenotypes, such as internodal distances (Dudley and Schmitt 1996), are required to link specific characters with neighbor effects.

Second, a detailed study of Impatiens population structure is necessary to determine the evolutionary consequences of kin selection within local patches. Impatiens occurs in groups that vary in size from a few individuals to hundreds of thousands. The interactions among plants that have been the focus of this study are highly localized within these larger groups. Thus, Impatiens is charac- 
terized by a two-level population structure. The dynamics of kin selection in hierarchically structured populations depend on the relative spatial scales of neighbor interaction, mating, dispersal, and density regulation (Kelly 1992, 1994a, 1994b; Taylor 1992a, 1992b; Wilson et al. 1992; Queller 1994). Each of these factors will need to be investigated in order to develop quantitative predictions for the magnitude and direction of selection within an Impatiens metapopulation.

\section{Phenotypic Plasticity and the Measurement of Kin Selection}

The effects of neighbor phenotypes on focal plant fitness seem to be mediated through the growth and development of focal plants. In p1 in 1993, neighbor manipulation significantly increased focal plant height and leaf number as well as focal plant seed production (table 1). Similar effects were observed in the 1994 experiments. These results are consistent with other studies on Impatiens that have shown plastic growth in response to differences in neighbor density (Weiner et al. 1990; Weiner and Thomas 1992). The differential growth of focal plants probably results from the manipulation of neighbors altering the amount and spectral quality of light incident on focal plants (Schmitt and Wulff 1993; Dudley and Schmitt 1996).

Plastic phenotypic response to neighbor phenotypes has an important general implication for field studies of kin selection in plant populations. It suggests that phenotypic regression methods for studying selection, such as contextual analysis (e.g., Heisler and Damuth 1987; Stevens et al. 1995), may not be able to detect an important class of neighbor effects on fitness. Contextual analysis is an extension of the multivariate phenotypic regressions methods for studying individual selection (Lande and Arnold 1983). In a contextual analysis, the set of variables used to predict fitness is expanded to include group phenotypic values as well as individual phenotypic values in the multiple regression (Heisler and Damuth 1987). Goodnight et al. (1992) claim that a significant partial regression of fitness onto a group-level character indicates that group-level selection is occurring.

The difficulty with this method suggested by the present study is that individual phenotypic values may not be simple properties of individual plants. This point is contrary to the basic quantitative genetic model that underlies phenotypic selection analyses. As described above, the basic model assumes that the phenotype of an individual is determined by its genotypic value plus a random error (Lande 1979). However, when there is phenotypic plasticity, as is very common in plants, the phenotype of an organism may be determined not only by its own genotype but also by the genotypes of its neighbors. In this case, a significant regression of fitness onto an individual trait value may due to differences among plants in the genotypes of their neighbors and not to differences in the genotypes of the plants themselves.

This possibility can be illustrated with the data from $\mathrm{p} 1$ in 1993. The ANOVA for fruit production revealed a strong and highly significant effect of neighbor treatment (fig. 1). However, if we reanalyze the data using an ANCOVA and include focal plant leaf number as a covariate, the neighbor treatment effect disappears $(F=0.38, \mathrm{df}=1,14, P=.55)$. In contrast, the intraclass regression of fruit production onto focal plant leaf number is highly significant $(F=9.88$, 
df $=1,14, P<.007)$ and explains most of the variation that was accounted for by the neighbor treatment in the previous ANOVA. Thus, if we were to evaluate the relative importance of group and individual effects on fitness from the ANCOVA, we would conclude that the neighbor phenotype is unimportant. We fail to detect a strong neighbor effect because it is subsumed within the individual phenotypic value. Stated another way, the causal pathway from neighbor phenotype to plant fitness passes through the individual phenotype (fig. 5).

This observation bears on contextual analysis in that the simple comparison of the strength of association between fitness and individual (leaf number) versus group (manipulated or unmanipulated) phenotypes derived from the ANCOVA results is directly analogous to a comparison of group and individual selection gradients in a contextual analysis. It is thus not clear that a contextual analysis of unmanipulated plants would have revealed the results of this study. Returning to the path diagram (fig. 5), we find that contextual analysis will pick up the direct neighbor effect on focal plant fitness (path $h$ ) but may miss the indirect path (path $f$ to path $b$ ). This point provides additional support for the notion that manipulative studies are a desirable complement to correlative studies of natural selection (Mitchell-Olds and Shaw 1987; Wade and Kalisz 1990).

\section{ACKNOWLEDGMENTS}

This article benefited from reviews by A. Molumby, N. Johnson, E. Simms, D. Thiede, L. Horth, G. Schott, B. Black, S. Kalisz, M. Liebold, M. Wade, K. Holsinger, and four anonymous reviewers. A. Pringle, M. Prynne, D. Franke, C. Grimsley, A. Molumby, and M. Wade assisted with the fieldwork. S. Kalisz, D. Theide, and S. Tonsor provided logistical assistance. The Kellogg Bird Stactuary and Kellogg Experimental Forest allowed me access to their facilities. I am also indebted to M. Amba and her colleagues at the Oxoboxo Institute for Animal Dermatology for their continual input. I received personal financial support from a National Science Foundation predoctoral fellowship and a Genetics Training Grant and research funding from $\mathrm{M}$. Wade and a Hinds fund grant.

\section{LITERATURE CITED}

Allard, R. W., and J. Adams. 1969. Population studies in predominantly self-pollinating species. XIII. Intergenotypic competition and population structure in barley and wheat. American Naturalist 103:621-645.

Antonovics, J., and D. A. Levin. 1980. The ecological and genetic consequences of density-dependent regulation in plants. Annual Review of Ecology and Systematics 11:411-452.

Argyres, A. Z., and J. Schmitt. 1991. Microgeographic genetic structure of morphological and life history traits in a natural population of Impatiens capensis. Evolution 45:178-189.

Arnold, S. J., and M. J. Wade. 1984a. On the measurement of natural and sexual selection: theory. Evolution 38:709-719.

- 1984b. On the measurement of natural and sexual selection: applications. Evolution 38: 720-734.

Breden, F., and M. J. Wade. 1989. Selection within and between groups of the imported willow leaf beetle. American Naturalist 134:35-50.

Cheverud, J. M. 1985. A quantitative genetic model of altruistic selection. Behavioral Ecology and Sociobiology 16:239-243. 
Cheverud, J. M., and A. J. Moore. 1995. Quantitative genetics and the role of the environment provided by relatives in behavioral evolution. In C. Boake, ed. Quantitative genetic studies of behavioral evolution. University of Chicago Press, Chicago.

Dudley, S. A., and J. Schmitt. 1995. Genetic differentiation in morphological responses to simulated foliage shade between populations of Impatiens capensis from open and woodland sites. Functional Ecology 9:655-666.

1996. Testing the adaptive plasticity hypothesis: density dependent selection on manipulated stem length in Impatiens capensis. American Naturalist 147:445-465.

Falconer, D. S. 1981. Introduction to quantitative genetics. Wiley, New York.

Geber, M. A. 1989. Interplay of morphology and development on size inequality: a polygonum greenhouse study. Ecological Monographs 59:267-288.

Goldberg, D. E. 1987. Neighborhood competition in an old-field plant community. Ecology 68: 1211-1223.

Goodnight, C. J. 1985. The influence of environmental variation on group and individual selection in a cress. Evolution 39:545-558.

Goodnight, C. J., J. M. Schwartz, L. Stevens. 1992. Contextual analysis of models of group selection, soft selection, hard selection and the evolution of altruism. American Naturalist 140: $743-761$.

Govindaraju, D. R. 1988. Mating systems and the opportunity for group selection in plants. Evolutionary Trends in Plants 2:99-106.

Grafen, A. 1985. A geometrical view of relatedness. Oxford Surveys in Evolutionary Biology 2:29-89.

Griffing, B. 1967. Selection in reference to biological groups. I. Individual and group selection applied to populations of unordered groups. Australian Journal of Biological Sciences 20:127-139. 1989. Genetic analysis of plant mixtures. Genetics 122:943-956.

Hamilton, W. D. 1964a. The genetical evolution of social behavior. I. Journal of Theoretical Biology $7: 1-16$.

1964b. The genetical evolution of social behavior. II. Journal of Theoretical Biology 7:17-52.

1975. Innate social aptitudes of man: an approach to evolutionary genetics. Pages 133-155

in R. Fox, ed. Biosocial anthropology. Wiley, New York.

Harper, J. L. 1977. Plant population biology. Academic Press, New York.

Heisler, L., and J. Damuth. 1987. A method for analyzing selection in hierarchically structured populations. American Naturalist 130:582-602.

Horn, H. 1971. The adaptive geometry of trees. Princeton University Press, Princeton, N.J.

Kalisz, S. 1986. Variable selection on the time of germination in Collinsia verna. Evolution 40: 479-491.

Kelly, J. K. 1992. Restricted migration and the evolution of altruism. Evolution 46:1492-1495.

1994a. The effect of scale dependent processes on kin selection: mating and density regulation. Theoretical Population Biology 46:32-57.

$1994 b$. The evolution of communal foraging in hierarchically structured populations. Behavioral Ecology and Sociobiology 35:205-212.

Knight, S. E., and D. Waller. 1987. Genetic consequences of outcrossing in the cleistogamous annual, Impatiens capensis. I. Population genetic structure. Evolution 41:969-978.

Lande, R. 1979. Quantitative genetic analysis of multivariate evolution applied to brain:body allometry. Evolution 33:402-416.

Lande, R., and S. Arnold. 1983. The measurement of selection on correlated characters. Evolution 37:1210-1236.

Levin, D. A. 1988. Local differentiation and the breeding structure of plant populations. In L. D. Gottleib and S. K. Jain, eds. Plant evolutionary biology. Chapman \& Hall, New York.

Lynch, M. 1987. Evolution of intrafamily interactions. Proceedings of the National Academy of Sciences of the USA 84:8507-8511.

McCauley, D. 1994. Intrademic group selection imposed by a parasitoid-host interaction. American Naturalist 144:1-13.

Mitchell-Olds, T. 1987. Analysis of local variation in plant size. Ecology 68:82-87.

Mitchell-Olds, T., and J. Bergelson. 1990a. Statistical genetics of an annual plant, Impatiens capensis. I. Genetic basis of quantitative variation. Genetics 124:407-415. 
1990b. Statistical genetics of an annual plant, Impatiens capensis. II. Natural selection. Genetics 124:417-421.

Mitchell-Olds, T., and R. G. Shaw. 1987. Regression analysis of natural selection, statistical inference, and biological interpretation. Evolution 41:1149-1161.

Pacala, S. W., and J. A. Silander. 1985. Field tests of neighborhood population dynamic models of two annual weed species. Ecological Monographs 60:113-134.

Price, G. R. 1970. Selection and covariance. Nature (London) 277:520-521.

Queller, D. C. 1992. Quantitative genetics, inclusive fitness and group selection. American Naturalist 139:540-558.

1994. Genetic relatedness in a viscous population. Evolutionary Ecology 8:70-73.

Robertson, A. 1966. A mathematical model of the culling process in dairy cattle. Animal Productivity 8:93-108.

Schemske, D. W. 1984. Population structure and local selection in Impatiens pallida, a selfing annual. Evolution 38:817-832.

Schmitt, J., and R. D. Wulff. 1993. Light spectral quality, phytochrome, and plant competition. Trends in Ecology \& Evolution 8:47-51.

Schmitt, J., D. Ehrhardt, and D. Swartz. 1985. Differential dispersal of self-fertilized and outcrossed progeny in jewelweed (Impatiens capensis). American Naturalist 126:570-575.

Schmitt, J., J. Eccleston, and D. Ehrhardt. 1987. Dominance and suppression, size-dependent growth, and self-thinning in a natural Impatiens capensis population. Journal of Ecology 75:651-665.

Schoen, D. J., and R. G. Latta. 1989. Spatial autocorrelations of genotypes in populations of Impatiens pallida and Impatiens capensis. Heredity 63:181-189.

Schoen, D. J., G. Bell, and M. J. Lechowicz. 1994. The ecology and genetics of fitness in forest plants. IV. Quantitative genetics of fitness components in Impatiens pallida. American Journal of Botany 81:232-239.

Sokal, R. R., and F. J. Rohlf. 1981. Biometry. W. H. Freeman, New York.

Stevens, L., C. Goodnight, and S. Kalisz. 1995. Multi-level selection in natural populations of Impatiens capensis. American Naturalist 145:513-526.

Stewart, S. C., and D. J. Schoen. 1987. Pattern of phenotypic viability and fecundity selection in a natural population of Impatiens pallida. Evolution 41:1290-1301.

Stratton, D. A. 1992. Life cycle components of selection in Erigeron annuus. I. Phenotypic selection. Evolution 46:92-106.

Taylor, P. D. 1992a. Altruism in viscous populations: an inclusive fitness model. Evolutionary Ecology 6:352-356.

$1992 b$. Inclusive fitness in a homogeneous environment. Proceedings of the Royal Society of London B, Biological Sciences 249:299-302.

Uyenoyama, M. K., M. W. Feldman, and L. D. Mueller. 1981. Population genetic theory of kin selection: multiple alleles at one locus. Proceedings of the National Academy of Sciences of the USA 78:5036-5040.

Wade, M. J. 1980. Kin selection: its components. Science (Washington, D.C.) 210:665-667.

1985. Hard selection, soft selection, kin selection, and group selection. American Naturalist 125:61-73.

Wade, M. J., and F. J. Breden. 1987. Kin selection in complex groups: mating structure, migration structure, and the evolution of social behaviors. Pages 273-283 in B. D. Chepko-Sade and Z. T. Halpin, eds. Mammalian dispersal patterns: the effects of social structure on population genetics. University of Chicago Press, Chicago.

Wade, M. J., and S. Kalisz. 1990. The causes of natural selection. Evolution 44:1947-1955.

Waller, D. 1985. The genesis of size hierarchies in seedling populations of Impatiens capensis. New Phytologist 100:243-260.

Weiner, J., G. M. Berntson, and S. C. Thomas. 1990. Competition and growth form in a woodland annual. Journal of Ecology 78:459-469.

Weiner, J., and S. C. Thomas. 1992. Competition and allometry in three species of annual plants. Ecology 73:648-656.

Williams, G. C., and D. C. Williams. 1957. Natural selection of individually harmful social adaptations among sibs with special reference to social insects. Evolution 11:32-39. 
Willham, R. L. 1963. The covariance between relatives for characters composed of components contributed by related individuals. Biometrics 19:18-27.

Wilson, D. S. 1975. A theory of group selection. Proceedings of the National Academy of Sciences of the USA 72:143-146.

1980. The natural selection of populations and communities. Benjamin/Cummings, Menlo Park, Calif.

Wilson, D. S., L. A. Dugatkin, and G. Pollock. 1992. Can altruism evolve in purely viscous populations? Evolutionary Ecology 6:331-341.

Wilson, E. O. 1975. Sociobiology. Harvard University Press, Cambridge, Mass.

Wright, S. 1968. Evolution and genetics of populations. Vol. 1. Genetic and biometric foundations. University of Chicago Press, Chicago.

Associate Editor: Kent E. Holsinger 